\title{
Emission line objects in NGC 6822. New planetary nebula candidates ${ }^{\star} \star \star \star$
}

\author{
L. Hernández-Martínez and M. Peña
}

\author{
Instituto de Astronomía, Universidad Nacional Autónoma de México, Apdo. Postal 70264, Méx. D. F., 04510 México \\ e-mail: [lhernand;miriam]@astroscu.unam.mx
}

Received 17 June 2008 / Accepted 6 November 2008

\section{ABSTRACT}

\begin{abstract}
Aims. Images obtained with the CTIO 4-m telescope and the MOSAIC-2 wide field camera in [O III] 5007 and $\mathrm{H} \alpha$ on-band and off-band filters are analyzed to search for emission line objects in the dwarf galaxy NGC 6822. In particular we search for planetary nebula (PN) candidates. In addition, imaging and spectroscopy of a sub-sample of objects obtained with the ESO VLT and FORS 2 spectrograph are used to calibrate the MOSAIC imaging.

Methods. In the continuum-subtracted images, a large number of line emission regions were detected, for which we measured instrumental magnitudes in all the filters. The [O III] 5007 and $\mathrm{H} \alpha+[\mathrm{N} \mathrm{II}]$ magnitudes were calibrated with the spectroscopy.

Results. Based upon some criteria to distinguish between PNe and compact HII regions, we have found 26 PN candidates, increasing the known sample by 8 objects. Also we detected a number of compact HII regions and about 20 stellar-like objects emitting in $\mathrm{H} \alpha$. For all the objects we present coordinates, instrumental magnitudes and nebular [O III] and $\mathrm{H} \alpha+[\mathrm{N}$ II] fluxes. The observed luminosity function for the PN [O III] 5007 magnitudes (PNLF) and the cumulative PNLF were calculated. We confirm that the PNLF presents a dip similar to the one detected for the Small Magellanic Cloud at 2.5 mag below the maximum. The cumulative PNLF returns a value $M_{5007}^{\star}=-3.71_{-0.42}^{+0.21}$ for the peak absolute magnitude of the PNLF which is faint (but within uncertainties) compared to the value expected for galaxies with a metallicity similar to that of NGC 6822. From our best fit to the observed PNLF we obtained a rough estimate of the distance modulus $m-M=23.64_{-0.43}^{+0.23}$ mag, which agrees within uncertainties with recent values from Cepheid stars reported in the literature. Also the number of PNe in the brightest $0.5 \mathrm{mag}$, normalized to the galactic bolometric luminosity, was estimated to be $\alpha_{0.5} \sim\left(3.8_{-071}^{+0.90}\right)$ E-9. This number is similar to the values derived for galaxies with recent star formation and small galaxies $\left(M_{\mathrm{B}}\right.$ fainter than $-18 \mathrm{mag}$ ) and larger than the values for early-type galaxies.
\end{abstract}

Key words. ISM: HII regions - ISM: planetary nebulae: general - galaxies: individual: NGC 6822 (DDO 209)

\section{Introduction}

Planetary nebulae (PNe) are valuable tracers of the stellar population of low and intermediate mass stars. Due to their selective emission in a small number of strong and narrow emission lines, they can be discovered at significant distances within the nearby Universe (at least $30 \mathrm{Mpc}$ ). Their study provides accurate information on the luminosity, age, metallicity, and dynamics of the parent stellar population. This makes them very useful to test a number of theories about the evolution of stars and galaxies.

$\mathrm{PNe}$ are also useful as distance indicators through the [O III] 5007 planetary nebulae luminosity function (PNLF). The advantage of this method is that we can see bright PNe in galaxies of all kind of Hubble types, and PNe are easily indentified. Jacoby (1989) and Ciardullo et al. (1987) reported that the PNLF had the same shape in all their investigated galaxies so it can be used as a standard candle, if we assume a complete sample in the 2 or 3 brightest magnitudes. However, there is evidence that the standard PNLF does not fit well in some galaxies. Jacoby \& De Marco (2002) show that the PNLF of the Small Magellanic Cloud presents a prominent dip 4 mag below the brightest PN.

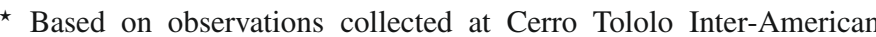
Observatory, National Optical Astronomy Observatory, which is operated by AURA, Inc., under cooperative agreement with the National Science Foundation.

$\star \star$ Based on observations collected at the European Southern Observatory, VLT, Paranal, Chile, program ID 077.B-0430.
}

Leisy et al. (2005), with 17 PN candidates, obtained a precarious PNLF for NGC 6822 which also presents a dip around 2 mag below the brightest PN. The main aim of this work is to investigate the PN populations in NGC 6822 by performing a deep survey for PN candidates.

NGC 6822 (DDO 209, IC 4895) is a nearby Local Group gas-rich dIrr galaxy. It is located at a distance modulus of $23.31 \pm 0.02$ (Cepheid distance by Gieren et al. 2006) from our galaxy; moving away at $V_{\text {rad }} \sim 44 \mathrm{~km} \mathrm{~s}^{-1}$ and at $880 \mathrm{kpc}$ from M 31 (Mateo 1998). Recently it has been shown that NGC 6822 possesses two components: a huge HI disk of about $6 \times 13 \mathrm{kpc}$, centered at the optical center, which includes the well-known optical bar and where the young stellar population resides, extending in zones with radii over $5 \mathrm{kpc}$ from the center (de Blok \& Walter 2000; de Blok \& Walter 2006), and a second component constituted by a spheroidal stellar structure as extensive as the HI disk, but with its major axis at roughly right angles to it. This spheroid contains a substantial intermediate-age population (red giants, AGB and carbon stars, Demers et al. 2006, and references therein).

This galaxy shows clear evidence for recent star formation with more than a hundred HII regions detected (Killen \& Dufour 1982; Hodge et al. 1988). Ford et al. (2002) found 7 candidate $\mathrm{PNe}$ and more recently Leisy et al. (2005) have reported several new PN candidates, increasing the total sample to 17, most of them located in the central zone. An additional PN was found by Richer \& McCall (2007). In the chemical context NGC 6822 
is a metal-poor galaxy, with an interstellar medium (ISM) abundance of about $0.2 Z_{\odot}$ (e.g., Lee et al. 2006; Richer \& McCall 2007). Spectroscopic studies of some emission line objects have been performed by Killen \& Dufour (1982), Richer \& McCall (1995, 2007), Peimbert et al. 2005; and others. From these studies several PN candidates have been confirmed as true PNe.

Historically this galaxy seems to have not been affected by tidal effects from the Milky Way or M31, thus it is suitable for chemical evolution studies. However, recent dynamical studies by de Blok \& Walter (2006), reported a "North West companion", and other peculiar structures which indicate that probably there were at least some encounters or tidal effects in this galaxy.

We obtained deep imaging of the whole face of NGC 6822 in an effort to extend the known PN population towards one or two magnitudes fainter. As part of the same program, a sub-sample of the detected PNe and HII regions has been studied spectroscopically to determine their chemical composition. First, we obtained on-band off-band images in [O III] $5007 \AA$ and $\mathrm{H} \alpha$, with wide field cameras, to search for emission line objects. We classified the objects according to the criteria proposed by Peña et al. (2007) and separated them into HII regions, PN candidates and $\mathrm{H} \alpha$-emission stellar objects. In this paper we present the results of the imaging and use the spectra to validate the identification of the objects as PNe or HII regions. The results of spectroscopy will be presented in a second paper where line ratios, fluxes, physical conditions and chemical compositions will be analyzed.

The paper is organized as follows: in Sect. 2 we present the observations and data reduction. In Sect. 3 comparative photometry and flux calibration for the detected objects are performed. The final samples and their distribution in the galaxy are presented in Sect. 4 and in Sect. 5 we discuss the differential PNLF, the cumulative PNLF, and the number of PNe normalized to the parent-galaxy bolometric luminosity, $\alpha_{0.5}$. Our results are summarized in Sect. 6.

\section{Observations and data reduction}

\subsection{Imaging}

NGC 6822 was observed on 2005 September 4 and October 9, using the CTIO 4-m Blanco telescope with the MOSAIC 2 camera. The NOAO CCD MOSAIC 2 is a wide field imager of $8192 \times 8192$ pixels, with a spatial scale of $0.27^{\prime \prime}$ per pix. This provides a field of view of $36.8^{\prime} \times 36.8^{\prime}$. The whole image is divided in $8 \mathrm{CCDs}$, each one with two amplifiers.

Due to the angular size of the galaxy (about $28^{\prime} \times 40^{\prime}$ if we include the distribution of faint $\mathrm{C}$ stars), it was possible to cover almost the entire galaxy with just one exposure of the MOSAIC 2 imager. This gave us the advantage of obtaining very homogeneous data in each filter. The center of the observed field is $\mathrm{RA}=19: 45: 00.20, \mathrm{Dec}=-14: 48: 51.1$. Filter characteristics and exposure times are listed in Table 1 . In order to cover the CCD gaps between the $8 \mathrm{CCDs}$ we took dithered exposures with the configuration $(64,126)$.

On 2006 August 20 and 22, we observed NGC 6822 with the ESO VLT UT1 (Antu) telescope, in Cerro Paranal, Chile. The FORS 2 (Focal Reducer Spectrograph 2) was used to obtain spectroscopic data to characterize the interstellar medium in NGC 6822 (program ID 077.B-0480). The wide field of FORS 2 $\left(6.8^{\prime} \times 6.8^{\prime}\right)$ was used to study two fields. The first one includes the central part of the galaxy centered at $\mathrm{RA}=19: 44: 55.5$, Dec $=-14: 48: 00.0$ and the second covers an outskirts zone in the north-west region of the galaxy centered in $\mathrm{RA}=19: 43: 59.2$,
Table 1. CTIO-MOSAIC (above) and VLT-FORS 2 (below) imaging characteristics.

\begin{tabular}{lccc}
\hline \hline Filter & $\lambda_{\mathrm{c}}(\AA)$ & $F W H M(\AA)$ & Exp. time $(\mathrm{s})$ \\
\hline$[\mathrm{O}$ III] & 4990 & 50 & $8 \times 1200$ \\
$\mathrm{D} 51$ & 5130 & 154 & $4 \times 900$ \\
$\mathrm{H} \alpha$ & 6563 & 80 & $2 \times 1200$ \\
$\mathrm{H} \alpha+8$ & 6650 & 80 & $2 \times 1200$ \\
\hline$[\mathrm{O} \mathrm{III]}]$ & 5045 & 59 & 960 \\
$V_{\text {Bess }}$ & 5540 & 1115 & 60 \\
$\mathrm{H} \alpha$ & 6604 & 64 & 960 \\
$R_{\text {special }}$ & 6550 & 1650 & 60 \\
\hline
\end{tabular}

Dec $=-14: 46: 14.9$. The pre-imaging observations of the program, obtained to select the candidates for a subsequent multiobject spectroscopic run, have been used here to corroborate the CTIO MOSAIC data. Filter characteristics and exposure times are presented in Table 1.

\subsection{Spectroscopy}

Spectrophotometric data for several PN candidates and compact HII regions were obtained with the ESO Very LargeTelescope FORS 2 in MXU mode, on 2006 August 20 and 21. The grisms 600B and 600RI were used to cover a spectral range from about 3600 to $7500 \AA$. The observed spectra are not affected by atmospheric dispersion because the linear atmospheric dispersion compensator (LADC) is automatically set when observing with VLT-FORS. This compensates completely for atmospheric dispersion effects when observing at zenith distances lower than $45^{\circ}$, which is our case. We obtained three frames for each grism, with exposure times of $1800 \mathrm{~s}$ for each 600B exposure and $1500 \mathrm{~s}$ for each 600RI. The slit width was $1^{\prime \prime}$ for all the objects and the spectral resolution varied from about $0.7 \AA$ to 1.2 A. Data were reduced and calibrated with the ESO pipeline and $\mathrm{IRAF}^{1}$ routines. The standard stars EG274, LDS749B and BMP16274 were observed through a slit of $5^{\prime \prime}$ width for flux calibration. In this paper we use the spectroscopic data to confirm the classification of some objects and to calibrate the instrumental [O III] 5007 and $\mathrm{H} \alpha$ magnitudes. During the spectroscopic run the sky was clear and the seeing conditions varied from 0.7 to $0.9^{\prime \prime}$, however some flux could have been lost in the $1^{\prime \prime}$ slit. The losses can amount to 15-20\% which should be considered in the uncertainties of our flux calibration. The [O III] and $\mathrm{H} \alpha$ spectroscopic fluxes are presented in Table 2. In a second paper we will present all the spectral data: line fluxes, ratios, and their analysis.

\subsection{Data reduction}

The CTIO MOSAIC 2 data were reduced using the MSCRED IRAF package. We followed the reduction procedure given by Jannuzi, Claver, and Valdes at http: //www . noao . edu/noao/ noaodeep/Reduction0pt/framesv6.html (Valdes 1998). We perform the cross talk correction, trimming, overscan, bias subtraction, flat field division, and the correction for bad pixels (bpm) and cosmic rays (crmask) as indicated in the package. The astrometric solution was found using the USNO-A2.0

\footnotetext{
1 IRAF is distributed by the National Optical Observatory, which is operated by the Associated Universities for Research in Astronomy, Inc., under contract to the National Science Foundation.
} 
Table 2. Characteristics of emission line objects in NGC 6822.

\begin{tabular}{|c|c|c|c|c|c|c|c|c|c|c|c|c|}
\hline obj No. & $\mathrm{RA}^{2}$ & $\operatorname{Dec}^{2}$ & $m_{i}(5007)$ & $m_{i}(\mathrm{D} 51)$ & $m_{i}(\mathrm{Ha})$ & $m_{i}(\mathrm{Ha} 8)$ & $\log F_{5007}^{3}$ & $\log F_{5007}^{4}$ & $\log F_{\mathrm{Ha}}^{3}$ & $\log F_{\mathrm{Ha}}^{5}$ & $5007 / \mathrm{Ha}$ & Other $\mathrm{ID}^{6}$, comments \\
\hline PN 1 & $19: 44: 35.45^{2}$ & $-14: 40: 50.5$ & 24.94 & 23.49 & 22.84 & 24.70 & 3 & $-15.73^{4}$ & & $-14.99^{5}$ & 0.18 & faint \\
\hline PN 2 & 19:45:56.38 & $-14: 40: 50.5$ & 22.55 & 24.35 & 22.32 & 25.00 & & -14.69 & & -14.76 & 1.18 & $\mathrm{PN}^{6}$ \\
\hline PN 3 & 19:45:02.70 & $-14: 41: 36.0$ & 24.50 & - & 22.80 & 24.70 & & -15.54 & & -14.97 & 0.27 & faint 5007 \\
\hline PN 4 & 19:45:01.53 & $-14: 41: 36.3$ & 21.05 & $>24.0$ & 20.99 & 23.30 & -13.96 & -14.03 & -14.12 & -14.17 & 1.39 & PN4, v \\
\hline PN 5 & 19:44:52.02 & $-14: 42: 18.0$ & 24.11 & $>25.0$ & 22.83 & $>25.0$ & & -15.37 & & -14.99 & 0.41 & $\mathrm{PN} 3, \mathrm{v}$ \\
\hline PN 6 & 19:44:02.29 & $-14: 42: 43.3$ & 21.52 & 23.37 & 21.71 & 24.70 & -14.19 & -14.24 & -14.46 & -14.49 & 1.80 & PN1, v \\
\hline PN 7 & 19:44:49.11 & $-14: 43: 00.6$ & 22.46 & 23.85 & 22.05 & $>25.0$ & & -14.65 & & -14.64 & 0.99 & PN2, v \\
\hline PN 8 & 19:46:02.22 & $-14: 43: 42.2$ & 22.90 & 24.71 & 22.16 & 24.70 & & -14.84 & & -14.69 & 0.70 & PN6 \\
\hline PN 9 & 19:44:58.77 & $-14: 44: 14.7$ & $>25.0$ & 23.70 & 22.26 & $>24.0$ & & -15.76 & & -14.74 & 0.09 & faint faint CS \\
\hline PN 10 & $19: 44: 56.93$ & $-14: 45: 18.6$ & 21.94 & 22.08 & 21.24 & $>25.0$ & -14.53 & -14.42 & -14.35 & -14.29 & 0.73 & PN19, v \\
\hline PN 11 & $19: 45: 00.74$ & $-14: 46: 28.9$ & 25.45 & $>25.5$ & 23.47 & 25.03 & & -15.96 & & -15.27 & 0.20 & faint \\
\hline PN 12 & 19:44:49.59 & $-14: 46: 31.3$ & 21.51 & $>24.0$ & 21.91 & $>24.0$ & -14.31 & -14.23 & -14.74 & -14.58 & 2.23 & PN14, v \\
\hline PN 13 & $19: 44: 31.27$ & $-14: 47: 14.9$ & 23.74 & 23.38 & 22.83 & $>24.0$ & & -15.21 & & -14.99 & 0.60 & PN15 \\
\hline PN 14 & 19:45:07.15 & $-14: 47: 31.4$ & 21.07 & 23.39 & 20.43 & 23.52 & -13.98 & -14.04 & -13.90 & -13.93 & 0.77 & PN7, v \\
\hline PN 15 & $19: 45: 00.59$ & $-14: 48: 04.6$ & $>25.3$ & $>25.0$ & 23.40 & 25.24 & & -15.87 & & -15.24 & - & no 5007 \\
\hline PN 16 & $19: 44: 49.52$ & $-14: 48: 03.5$ & 21.74 & 23.17 & 21.68 & 23.91 & -14.33 & -14.33 & -14.53 & -14.48 & 1.40 & PN13, v \\
\hline PN 17 & $19: 44: 59.73$ & $-14: 48: 07.2$ & 23.06 & $>25.5$ & 23.20 & $>24.0$ & & -14.91 & & -15.15 & 1.73 & PN10, v \\
\hline PN 18 & 19:44:51.79 & $-14: 48: 15.7$ & 24.59 & $>25.5$ & 23.29 & 24.71 & -15.60 & -15.58 & & -15.19 & 0.41 & PN11, v \\
\hline PN 19 & 19:45:06.44 & $-14: 48: 39.5$ & 24.80 & $>25.0$ & 23.73 & $>25.0$ & -15.56 & -15.67 & -15.41 & -15.38 & 0.51 & PN9, v \\
\hline PN 20 & $19: 45: 11.50$ & $-14: 48: 53.6$ & 22.61 & 22.24 & 21.97 & 23.14 & & -14.71 & & -14.61 & 0.78 & PN20, v \\
\hline PN 21 & $19: 44: 50.90$ & $-14: 49: 13.8$ & 23.39 & 23.48 & 22.93 & 24.10 & -15.16 & -15.06 & -15.32 & -15.03 & 0.94 & PN12, v \\
\hline PN 22 & $19: 44: 58.90$ & $-14: 49: 31.0$ & 24.80 & 23.85 & 23.67 & 24.60 & & -15.67 & & -15.35 & 0.48 & faint \\
\hline PN 23 & $19: 44: 44.22$ & $-14: 50: 46.4$ & 23.32 & $>25.0$ & 22.92 & $>25.0$ & & -15.02 & & -15.02 & 1.00 & PN17, v \\
\hline PN 24 & 19:44:45.59 & $-14: 52: 37.0$ & 23.53 & $>25.0$ & 24.25 & $>26.0$ & & -15.12 & & -15.61 & 3.12 & PN16 \\
\hline PN 25 & 19:45:13.36 & $-14: 54: 48.6$ & 25.44 & $>25.0$ & 24.15 & $>25.0$ & & -15.95 & & -15.57 & 0.41 & faint 5007 \\
\hline PN 26 & $19: 45: 07.84$ & $-14: 55: 50.3$ & $>26.0$ & $>25.0$ & 23.81 & $>25.0$ & & -16.20 & & -15.42 & - & no 5007 \\
\hline HII 1 & $19: 44: 30.74$ & $-14: 41: 37.8$ & 19.11 & 20.22 & 18.40 & 21.14 & & -13.18 & & -13.04 & 0.71 & \\
\hline HII 2 & 19:44:56.80 & $-14: 42: 07.9$ & $>25.0$ & 22.15 & 22.48 & - & & $<-15.7$ & & -14.83 & - & HII 21, dif, no CS \\
\hline HII 3 & $19: 44: 32.54$ & $-14: 42: 23.8$ & 23.75 & 22.58 & 21.74 & 23.37 & & 15.21 & & -14.50 & 0.20 & comp, no 5007 \\
\hline HII 4 & $19: 44.54 .52$ & $-14: 42: 44.4$ & 23.29 & 22.01 & 20.92 & 22.94 & & -15.01 & & -14.15 & 0.14 & HII 20, comp, no 5007 \\
\hline HII 5 & $19: 44: 52.31$ & $-14: 42: 50.6$ & $>24.5$ & 22.79 & 22.27 & 23.60 & & $<-15.5$ & & -14.74 & - & HII 22, dif, no5007 \\
\hline HII 6 & $19: 44: 52.14$ & $-14: 42: 58.1$ & $>24.5$ & 22.81 & 22.11 & 23.48 & & $<-15.5$ & & -14.67 & - & HII 23, dif, \\
\hline HII 7 & $19: 44: 53.99$ & $-14: 43: 12.5$ & 21.99 & 20.85 & 21.28 & 21.83 & & -14.44 & & -14.30 & 0.72 & dif, faint \\
\hline HII 8 & $19: 44: 48.41$ & $-14: 43: 58.6$ & 21.57 & 21.37 & 22.03 & 22.61 & & -14.26 & & -14.63 & - & ght star \\
\hline HII 9 & $19: 44: 58.50$ & $-14: 44: 45.7$ & $>24.5$ & 23.07 & 21.76 & $>25.0$ & & -15.40 & & -14.51 & - & , faint 5007 \\
\hline HII 10 & 19:44:13.81 & $-14: 44: 46.9$ & $>24.5$ & 22.59 & 21.66 & 23.96 & & $<-15.3$ & & -14.47 & - & dif, no 5007 \\
\hline HII 11 & $19: 45: 17.09$ & $-14: 45: 29.5$ & 22.01 & 21.70 & 20.23 & 22.48 & & -14.45 & & -13.84 & 0.24 & dif, faint 5007 \\
\hline HII 12 & $19: 45: 01.58$ & $-14: 46: 05.6$ & $>25.0$ & $>25.0$ & 23.07 & 24.07 & & $<-16.0$ & & -15.09 & - & dif, \\
\hline HII 13 & 19:44:48.69 & $-14: 46: 15.0$ & 23.24 & 21.79 & 23.35 & 23.00 & & -14.99 & & -15.21 & - & dif, bright star \\
\hline HII 14 & 19:44:32.99 & $-14: 47: 31.6$ & 19.08 & 21.05 & 18.67 & 21.82 & & -13.17 & & -13.16 & 0.97 & KD_S5, comp \\
\hline HII 15 & 19:44:57.15 & $-14: 47: 50.0$ & 20.06 & 21.74 & 19.08 & 22.14 & -13.60 & -13.57 & -13.58 & -13.34 & 0.55 & HII 08 \\
\hline HII 16 & $19: 44: 30.88$ & $-14: 48: 26.3$ & 19.00 & 20.58 & 18.30 & - & & -13.13 & & -12.99 & 0.72 & KD_S2, comp \\
\hline HII 17 & 19:44:55.79 & $-14: 50: 31.3$ & $>24.5$ & 21.75 & 22.07 & 22.55 & & $<-15.3$ & & -14.65 & - & dif, no 5007 \\
\hline HII 18 & $19: 44: 42.57$ & $-14: 50: 32.2$ & 21.35 & 21.72 & 20.34 & 22.29 & & -14.16 & & -13.89 & 0.53 & KD_S10, comp \\
\hline HII 19 & $19: 44: 50.60$ & $-14: 52: 29.8$ & $>24.5$ & 22.12 & 23.17 & 23.27 & & -15.13 & & -15.13 & - & dif \\
\hline HII 20 & $19: 44: 50.80$ & $-14: 52.53 .5$ & 19.33 & 20.77 & 19.01 & 21.75 & & -13.28 & & -13.30 & 1.06 & comp \\
\hline HII 21 & $19: 45: 10.37$ & $-14: 54: 30.4$ & $>24.5$ & 22.49 & 21.13 & 22.96 & & -15.30 & & -14.24 & - & comp, faint 5007 \\
\hline HII 22 & 19:45:08.44 & $-14: 57: 30.6$ & $>24.5$ & 23.17 & 22.34 & 23.98 & & -15.62 & & -14.77 & - & dif, faint \\
\hline HII- 23 & 19:45:04.60 & $-14: 57: 31.7$ & 20.71 & 20.90 & 19.34 & 21.77 & & -13.88 & & -13.45 & 0.37 & comp \\
\hline HII 24 & $19: 45: 12.90$ & $-14: 57: 41.4$ & $>25.0$ & 22.71 & 23.30 & 24.04 & & $<-15.8$ & & -15.19 & - & dif, faint CS \\
\hline HII 25 & $19: 45: 13.90$ & $-14: 58: 47.2$ & 22.65 & 21.40 & 21.32 & 22.38 & & -14.73 & & -14.32 & 0.39 & dif \\
\hline Ha 1 & $19: 45: 59.21$ & $-14: 39: 37.6$ & 25.80 & 23.89 & 23.56 & 24.70 & & -16.11 & & -15.30 & 0.16 & faint PN? \\
\hline Ha 2 & $19: 44: 39.10$ & $-14: 41: 25.7$ & 25.68 & 23.65 & 23.87 & 23.74 & & -16.06 & & -15.44 & 0.24 & \\
\hline Ha 3 & $19: 44: 40.62$ & $-14: 42: 40.7$ & 23.94 & 22.30 & 22.75 & 23.16 & & -15.30 & & -14.95 & 0.45 & \\
\hline Ha 4 & $19: 45: 05.02$ & $-14: 44: 14.8$ & 24.59 & 22.95 & 23.14 & 24.13 & & -15.58 & & -15.12 & 0.35 & \\
\hline Ha 5 & $19: 45: 07.51$ & $-14: 44: 22.2$ & 24.04 & 22.70 & 21.22 & 23.35 & & -15.34 & & -14.28 & 0.09 & \\
\hline Ha 6 & $19: 45: 01.36$ & $-14: 44: 23.9$ & 23.78 & 22.70 & 21.97 & 22.53 & & -15.23 & & -14.61 & 0.24 & \\
\hline Ha 7 & $19: 44: 54.04$ & $-14: 46: 20.0$ & 23.37 & 21.85 & 22.47 & 22.82 & & -15.05 & & -14.83 & 0.60 & \\
\hline Ha 8 & 19:45:07.95 & $-14: 47: 05.6$ & 24.29 & 22.73 & 21.90 & 22.66 & & -15.45 & & -14.58 & 0.13 & \\
\hline Ha 9 & $19: 45: 17.50$ & $-14: 47: 27.6$ & 23.52 & 22.01 & 21.77 & 22.49 & & -15.11 & & -14.52 & 0.26 & \\
\hline На 10 & $19: 45: 07.24$ & $-14: 48: 14.3$ & 24.70 & 22.96 & 23.07 & 23.77 & & -15.63 & & -15.09 & 0.29 & \\
\hline Ha 11 & $19: 45: 28.73$ & $-14: 49: 05.6$ & 24.28 & 22.93 & 23.48 & 24.20 & & -15.44 & & -15.27 & 0.67 & faint PN? \\
\hline
\end{tabular}


Table 2. continued.

\begin{tabular}{|c|c|c|c|c|c|c|c|c|c|c|c|c|}
\hline Obj No. & $\overline{\mathrm{RA}^{2}}$ & 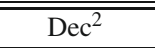 & $\overline{m_{i}(5007)}$ & $\overline{m_{i}(\mathrm{D} 51)}$ & 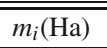 & $\overline{m_{i}(\mathrm{Ha} 8)}$ & $\overline{l o g} F_{5007}^{3}$ & $\overline{l o g} F_{5007}^{4}$ & 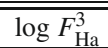 & $\overline{\log F_{\mathrm{Ha}}^{5}}$ & 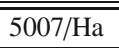 & other $\mathrm{ID}^{6}$, comments \\
\hline Ha 12 & $19: 44: 50.12$ & $-14: 50: 35.2$ & 24.00 & 22.42 & 23.05 & 23.47 & & -15.32 & & -15.08 & 0.57 & \\
\hline На 13 & $19: 45: 14.11$ & $-14: 51: 09.9$ & 23.05 & 21.60 & 22.09 & 22.60 & & -14.91 & & -14.66 & 0.57 & \\
\hline Ha 14 & $19: 45: 50.20$ & $-14: 51: 11.3$ & 25.44 & 23.97 & 23.18 & 23.40 & & -15.95 & & -15.14 & 0.15 & \\
\hline Нa 15 & $19: 45: 51.05$ & $-14: 51: 23.3$ & 26.44 & 24.00 & 23.36 & 23.79 & & -16.39 & & -15.22 & 0.07 & \\
\hline Ha 16 & $19: 45: 12.08$ & $-14: 52: 08.6$ & 23.52 & 22.04 & 21.77 & 22.30 & & -15.11 & & -14.52 & 0.26 & \\
\hline Ha 17 & $19: 45: 01.90$ & $-14: 52: 55.9$ & 24.58 & 23.07 & 23.51 & 24.16 & & -15.58 & & -15.29 & 0.51 & faint PN? \\
\hline Ha 18 & $19: 45: 08.30$ & $-14: 53: 22.0$ & 23.85 & 22.28 & 22.68 & 23.12 & & -15.26 & & -14.92 & 0.46 & \\
\hline На 19 & $19: 44: 55.91$ & $-14: 55: 06.4$ & 24.28 & 22.67 & 23.14 & 23.37 & & -15.44 & & -15.12 & 0.47 & \\
\hline Ha 20 & $19: 45: 04.16$ & $-14: 55: 57.4$ & $>26.0$ & 23.89 & 24.21 & 25.41 & & -16.20 & & -15.59 & 0.25 & \\
\hline Ha 21 & $19: 45: 14.85$ & $-14: 58: 37.2$ & 25.44 & 23.70 & 23.94 & 24.54 & & -15.95 & & -15.47 & 0.33 & faint PN? \\
\hline
\end{tabular}

${ }^{2}$ Coordinates for equinox 2000; ${ }^{3}$ flux from spectroscopy, in $\mathrm{erg} \mathrm{cm}^{-2} \mathrm{~s}^{-1}$. $\mathrm{Ha}=\mathrm{H} \alpha+[\mathrm{NII}] ;{ }^{4}$ flux calculated from the equation in Fig. 3a, in erg cm${ }^{-2} \mathrm{~s}^{-1} ;{ }^{5}$ flux calculated from the equation in Fig. 3b, in erg cm${ }^{-2} \mathrm{~s}^{-1} ;{ }^{6}$ previous ID from Leisy et al. (2005), Richer \& McCall (2007) and Killen \& Dufour (1982).

catalogue. Afterwards we corrected by the tangent-plane projected, and subtracted the sky.

The dithered [O III] 5007, $\mathrm{H} \alpha$ images and their off-band images were respectively shifted and combined in a single $8 \mathrm{~K} \times 8 \mathrm{~K}$ image. Therefore, finally we have a $2 \mathrm{~h}$ exposure time image in [O III] 5007, a $1 \mathrm{~h}$ image in off-band [O III], and $1 \mathrm{~h}$ images for $\mathrm{H} \alpha$ and its continuum. Observing nights were not photometric and the calibration flux could not be done with the standard star, but differential photometry can be performed. With the combination of seeing conditions and instrumental set-up we obtained a FWHM of 5.5 pix in the $\mathrm{H} \alpha$ image, 4.9 pix in the $\mathrm{H} \alpha$ continuum (hereafter $\mathrm{Ha} 8$ ); 3.9 pix in [O III] 5007, and 3.9 pix in D51.

The ESO FORS 2 pre-imaging were reduced and calibrated through the normal procedures of the ESO pipeline and the spectroscopy was reduced as mentioned in the previous section.

Our deep on-band off-band CTIO and ESO images were subtracted to produce 2 difference images to detect emission line objects. The "blinking" technique also was used to identify objects. A number of extended and compact emitting regions were found. All the objects detected in the [O III] images were also detected in the $\mathrm{H} \alpha$ image, but additional stellar-like $\mathrm{H} \alpha$ emitting objects were also found. The complete sample has been marked on our CTIO H $\alpha$ image which is shown in Fig. 1. Different symbols represent different type of objects (PNe, HII regions and $\mathrm{H} \alpha$ stellar objects). In the next section we discuss the criteria for distinguishing them. ID charts of the new objects are presented in Fig. 7.

\section{Distinguishing among different types of emission line objects}

The way in which the PNe candidates were selected follows the prescriptions given in Peña et al. (2007). That is, PN candidates should be point-like objects (at the distance of NGC 6822, a 1 pc radius nebula would appear as a $0.4^{\prime \prime}$ size object, thus, stellarlike) and the central star should not be detected or it should be very faint as compared to the central stars in HII regions. This is considering that at the distance of NGC 6822 an O9.5-B0 V star (the faintest able of producing a low excitation HII region), possessing an absolute magnitude $M_{\mathrm{V}} \sim-3.90$, (Martins et al. 2005) has an apparent magnitude $V \sim 19.5 \mathrm{mag}$ and a PN central star is typically 2-2.5 mag fainter (Méndez et al. 1992). Certainly it could happen that a field star is projected on a nebula, complicating the classification. Only spectroscopy can help in such a situation (see the cases discussed by Richer \& McCall 2007).

The excitation degree, represented by the $\left[\begin{array}{ll}\mathrm{O} & \mathrm{III}\end{array}\right]$ $5007 / \mathrm{H} \alpha$ flux ratio, is a commonly used criterion to select PN candidates (e.g., Magrini et al. 2000; Ciardullo et al. 2002; Herrmann et al. 2008). It is based on the fact that usually the brightest PNe have $I(5007) / I(\mathrm{H} \alpha)$ larger than HII regions. Moreover, Ciardullo et al. (2002) showed that PNe inhabit a very distinctive region in the $[\mathrm{O}$ III] $-\mathrm{H} \alpha$ space, with the brightest PNe showing dereddened $I(5007) / I(\mathrm{H} \alpha) \geq 2$. Recently Herrmann et al. (2008) codified this criterion. In Sect. 3.2 we apply this criterion to our objects and discuss its validity for NGC 6822.

In this section we will use the central star criterion to separate PN candidates from other types of emission line nebula. To proceed we have performed a comparative photometry measuring the instrumental magnitudes in the different MOSAIC images: [O III] 5007, D51, $\mathrm{H} \alpha(\mathrm{Ha})$ and $\mathrm{H} \alpha+8$ (Ha8). The IRAF task digiphot.apphot.phot was used. An aperture of 7 pix radius (equivalent to $1.9^{\prime \prime}$ ) was used to integrate the magnitudes of objects and the sky was subtracted from a ring of $2^{\prime \prime}$ width around. The results are listed in Table 2, where we present the coordinates (Cols. 2 and 3) and instrumental magnitudes (Cols. 4-7) for all the compact emission line objects. Note that due to the band width of $\mathrm{H} \alpha$ filter, $\mathrm{m}(\mathrm{Ha})$ includes $\mathrm{H} \alpha$ and both [N II] $6548 \AA$ and $6583 \AA$ lines. The instrumental photometric errors associated with the photometry are presented in Table 3.

The on-band magnitudes are plotted versus the off-band ones in Figs. $2 \mathrm{a}$ and $2 \mathrm{~b}$. In these figures it is possible to select PN candidates (filled dots) as those nebulae possessing a faint or undetectable star, while most $\mathrm{HII}$ regions (open squares) and $\mathrm{H} \alpha$ stellar objects (small diamonds) have a much brighter $m$ (D51) or $m(\mathrm{H} 8)$. In Fig. 2a, $m(5007)$ vs. $m(\mathrm{D} 51)$, the emission nebulae (PNs and HIIs) show intense $m(5007)$ magnitude while the $\mathrm{H} \alpha$-emitting objects behave as normal stars. Some HII regions are brighter than PN candidates and all of them have brighter central stars. The two PNe, with a bright central star at $m(\mathrm{D} 51) \sim 22 \mathrm{mag}$, are PN10 and PN20 in our list and they correspond to the previously known PN19 and PN20 for which Richer \& McCall (2007) showed that a field star is projected on these nebulae. In Fig. 2b, $m(\mathrm{Ha})$ vs. $m(\mathrm{Ha} 8)$, the gap in stellar magnitudes between HIIs and PNe is not as clear as in Fig. 2a as there are three PNe presenting a bright stellar continuum. They are the objects PN4, PN14 and PN20 of our list. For PN20 we already mentioned that there is a star projected on the nebula; PN4 and PN14 are two very bright spectroscopically confirmed PNe 


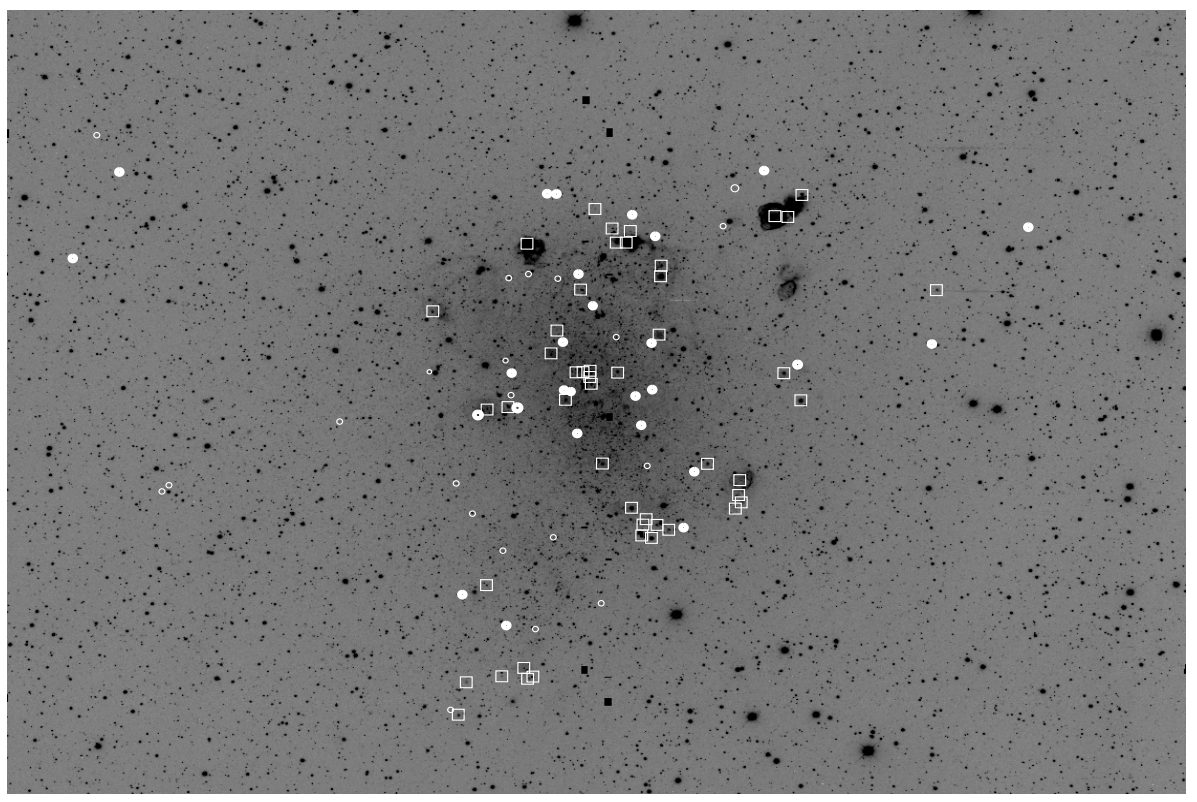

Fig. 1. Our CTIO MOSAIC $2 \mathrm{H} \alpha$ image. The field size is $36.8 \times 25.8 \operatorname{arcmin}(x-y)$. North is up, east is left. The objects of Table 2 have been marked as follows: big white circles represent PN candidates; open squares, HII regions; small white circles, $\mathrm{H} \alpha$ stellar objects. (See the text for a description of the selection mechanism). HII regions and $\mathrm{H} \alpha$ stellar objects are concentrated in the central zones in very good coincidence with the HI disk by de Blok \& Walter (2000) while PNe are more widely distributed.

Table 3. Photometric errors associated with instrumental magnitudes

\begin{tabular}{cccc}
\hline \hline mag & $\Delta m$ & mag & $\Delta m$ \\
\hline 19.000 & 0.007 & 23.000 & 0.057 \\
19.500 & 0.008 & 23.500 & 0.093 \\
20.000 & 0.009 & 24.000 & 0.117 \\
20.500 & 0.012 & 24.500 & 0.190 \\
21.000 & 0.015 & 24.800 & 0.215 \\
21.500 & 0.021 & 25.000 & 0.310 \\
22.000 & 0.027 & 25.500 & 0.530 \\
22.500 & 0.035 & 26.000 & 0.869 \\
\hline
\end{tabular}

(Hernández-Martínez \& Peña, in preparation) where apparently a sort of red star is projected on the nebula. In general HII regions are brighter in $\mathrm{H} \alpha$ than PN candidates, as expected due to their brighter central stars.

In Fig. 2a the $\mathrm{H} \alpha$ emitting stars closely follow the behavior of field stars, while in Fig. 2b they also follow the line of stars but at brighter $m(\mathrm{H} \alpha)$. The faintest among them could be low excitation PNe.

According to the results from Fig. 2, in Table 2 we have separated the objects by category: PN candidates, HII regions, stellar $\mathrm{H} \alpha$ emission-line objects.

\section{1. [O III] and $\mathrm{H} \alpha$ calibration}

A more confident way to distinguish between different types of emission line objects is by the spectral analysis of each object. However this is expensive in terms of telescope time because the objects are very faint. Our VLT spectroscopy included only 9 PNe and one compact HII region. The calibrated [O III] $5007 \AA$ and $\mathrm{H} \alpha+[\mathrm{N}$ II $] 6548,6583 \AA$ fluxes measured for these objects are listed in Cols. 8 and 10 of Table 2. Figures $3 \mathrm{a}$ and $3 \mathrm{~b}$ present the relation between our instrumental magnitudes and the logarithm of spectroscopic fluxes. It is notable that a linear correlation can be fitted to both sets of data with a very good correlation factor through $5 \mathrm{mag}$. These fits (equations shown in the figure caption) are used to calculate calibrated [O III] 5007 and $\mathrm{H} \alpha+[\mathrm{N} \mathrm{II}]$ fluxes for all the objects from their instrumental magnitudes (Cols. 9 and 11 of Table 2). The error bars in the fluxes (not shown in the table) include the errors in the
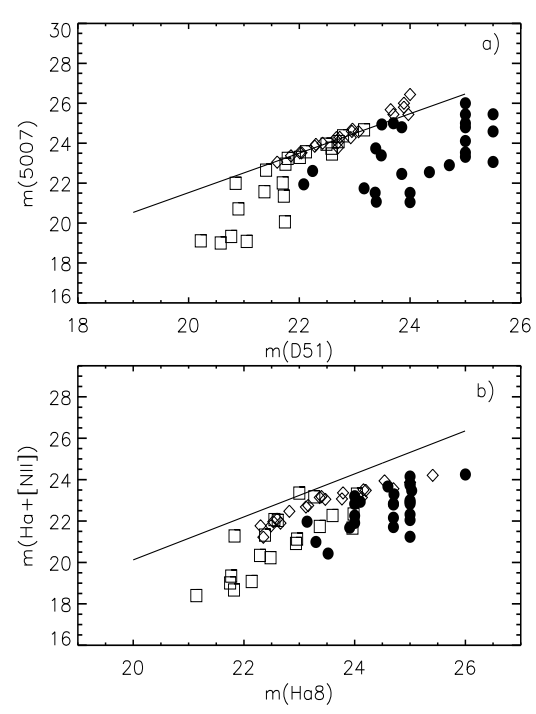

Fig. 2. On-band instrumental magnitudes are plotted versus the off-band magnitudes. The solid line presents the behavior of field stars (with no line emission). PN candidates are marked with fill dots, HII regions with open squares and $\mathrm{H} \alpha$ stellar objects as small diamonds. The accumulations of points at $m(\mathrm{D} 51)=25$ or 26 correspond to lower limits for $m$ (D51). In a) the $\mathrm{H} \alpha$ emission stars closely follow the behavior of field stars, while in b) they also follows the line of stars but at brighter $m(\mathrm{H} \alpha)$. The faintest among them could be low excitation PNe. Here the accumulation of points at $m(\mathrm{Ha} 8)=24,25$ and 26 is due to lower limits in $m(\mathrm{Ha} 8)$.

photometry (Table 3) plus an uncertainty of about $20 \%$ that should be added to the positive sign, due to possible losses in the spectroscopic slit (see Sect. 2). From the calibrated fluxes we have calculated the excitation degree [O III] $5007 / \mathrm{H} \alpha$ for our objects (Col. 12 in Table 2), which is discussed in the next section.

\subsection{The excitation degree of our emission line objects}

In Fig. 4 we plot the $\log$ of the dereddened [O III] $5007 /(\mathrm{H} \alpha+\mathrm{N}$ II]) flux ratio as a function of the absolute magnitude $M(5007)$ for PN candidates and HII regions of Table 2. To deredden the 

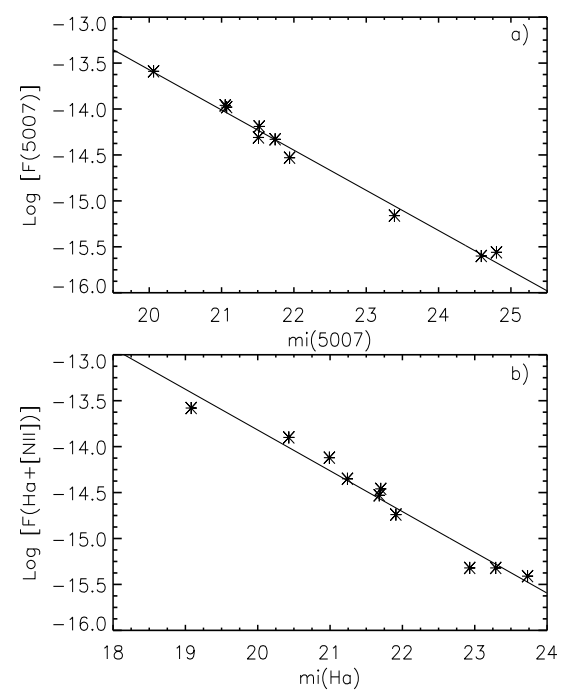

Fig. 3. The relations between the spectroscopic [O III] 5007 flux, $F(5007)$, and the instrumental magnitude $m_{\mathrm{i}}(5007)$ (upper panel) and between the spectroscopic flux $F(\mathrm{H} \alpha+[\mathrm{N}$ II] $)$ and the instrumental $m(\mathrm{Ha})$ for the spectroscopically analyzed objects (lower panel). The linear fits correspond to: $\log F(5007)=-\left(4.82+0.44 m_{\mathrm{i}}(5007)\right)$ in the upper panel (correlation coefficient $\left.R^{2}=0.95\right)$ and $\log F(\mathrm{H} \alpha+$ $[\mathrm{N} \mathrm{II}])=-\left(4.94+0.44 m_{\mathrm{i}}(\mathrm{Ha})\right)$ in the lower panel (correlation coefficient $R^{2}=0.97$ ).

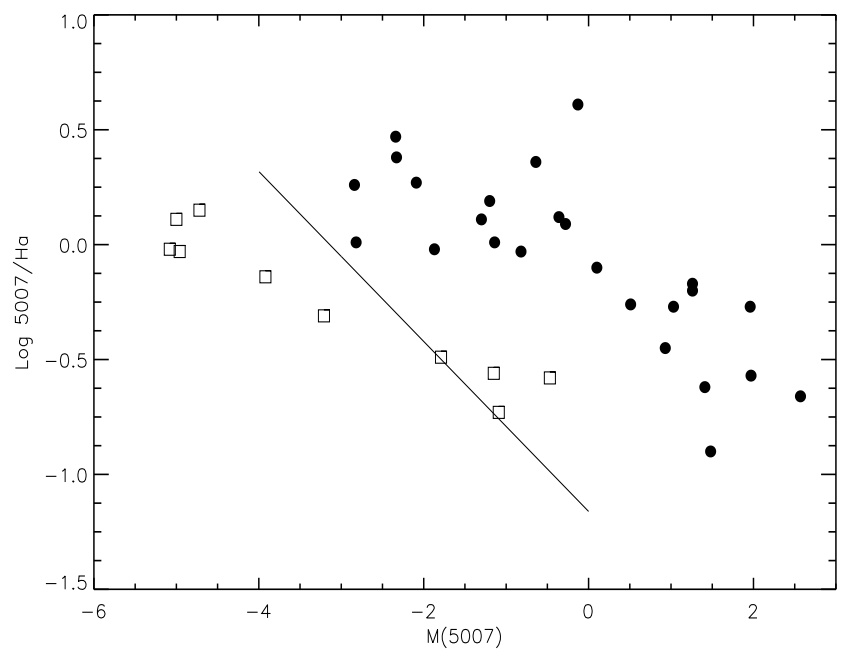

Fig. 4. The $\left[\begin{array}{ll}\mathrm{O} & \mathrm{III}\end{array}\right] / \mathrm{H} \alpha$ flux ratio vs. the absolute magnitude $M$ (5007) for PN candidates (black circles) and HII regions (open squares) in Table 2. The solid line corresponds to $\log 5007 / \mathrm{H} \alpha=$ $-0.37 M(5007)-1.16$, which is the lower limit suggested by Herrmann et al. (2998) for the zone occupied by PNe in the brightest 4 mag.

apparent fluxes we have considered for NGC 6822 a foreground extinction $E(B-V)=0.26 \pm 0.04 \mathrm{mag}$ (as derived for several authors, e.g., Massey et al. 1995; Gallart et al. 1996; Mateo 1998) and the reddening law by Fitzpatrick (1999). The Cepheid distance modulus, $23.31 \pm 0.02$, was used to calculate the absolute magnitudes. The solid line represents the lower limit of the zone proposed by Ciardullo et al. (2002), and computed by Herrmann et al. (2008), as representative for PNe in the brightest $4 \mathrm{mag}$, that is, the line corresponds to the equation: $\log 5007 / \mathrm{H} \alpha>-0.37 M(5007)-1.16$.

In this figure, it is evident that at a given $I(5007) / I(\mathrm{Ha})$ the brightest objects are HII regions (as expected due to the larger number of ionizing photons of HII region central stars). At a given $M(5007)$, $\mathrm{PNe}$ always have larger $5007 / \mathrm{H} \alpha$ ratios than HII regions and a ratio of 1.2 seems a safe value for most of the brightest PNe. All the PN candidates in our sample are safely above the limit by Herrmann et al., but our brightest PN (PN10 in Table 2), which is a confirmed type I PN with very intense [N II] (Richer \& McCall 2007; Hernández-Martínez \& Peña in preparation) has a $I(5007) / I(\mathrm{H} \alpha+\mathrm{N}$ II] $)$ ratio lower than one due to the $[\mathrm{N}$ II] line emission. Therefore, an excitation-degree criterion requering 5007/H $\alpha>1.6$, such as the one suggested by Ciardullo et al. (2002), would be discriminating against type I PNe.

In the faint zone of Fig. 4, where $M(5007)>-2.0$, all PN candidates fulfill the criterion proposed by Herrmann et al. (2008) but HII regions also fulfill this criterion. However HII regions can be easily separated from PN candidates because they do not fulfill the other criteria: they do not have a point-like appearance (at the distance of NGC 6822 many HII regions are diffuse) or they have a clearly visible central star. Then, for NGC 6822, the limit proposed by Herrmann et al. helps to distinguish PNe from HII regions only in the 2 brightest magnitudes. For fainter objects the faintness of the central star criterion is a better option.

\section{The final samples and their distribution}

Finally, from our deep search, we have a number of stellar and compact emitting objects which have been classified as PN candidates, HII regions and other stellar $\mathrm{H} \alpha$ emitting objects (from the characteristics of the latter, they could be Ae-Be stars and a few could be low excitation PN) which are presented in Table 2. The list includes coordinates, instrumental [O III] 5007, D51, $\mathrm{H} \alpha+[\mathrm{N}$ II $]$ and $\mathrm{Ha} 8$ magnitudes, the [O III $]$ and $\mathrm{H} \alpha+[\mathrm{N}$ II] fluxes for objects spectroscopically observed, the fluxes derived from the calibration by using equations in Figs. $3 \mathrm{a}$ and $3 \mathrm{~b}$, and the excitation degree $([\mathrm{O} \mathrm{III}] / \mathrm{Ha})$. In the last column we present previous IDs and comments.

There are 26 PN candidates, which is the largest sample obtained for NGC 6822 so far. The PN confirmed by spectroscopy (14 objects) are marked with a $\mathrm{v}$ in the last column of Table 2. We rediscovered all the $17 \mathrm{PN}$ candidates reported by Leisy et al. (2005), the PN found by Richer \& McCall (2007) and all their HII regions.

The distribution of emitting objects, differentiated by category, are shown in Fig. 1. Interestingly, the samples are concentrated mainly in the central zones of the galaxy (coinciding with the optical bar and the main body of the HI disk reported by de Blok \& Walter 2000), but HII regions and $\mathrm{H} \alpha$ emitting objects are more concentrated than PN candidates. This indicates that the star-forming zones (characterized by HII regions) are very centrally concentrated with no recent star formation farther than a few kpc from the center. This result was also found by de Blok \& Walter (2006). On the other hand, a few PN candidates are located at much larger galactocentric distance. Possibly some of these PNe belong to the intermediate-age population of the spheroidal distribution described by Demers et al. (2006). There are large zones in the outer regions of the galaxy, in particular towards the south-west, where no PN has been found. This is mainly due to the low stellar density in this zone.

\section{The PN sample and the planetary nebulae luminosity function (PNLF)}

For our 26 PN candidates we determined [O III] 5007 and $\mathrm{H} \alpha+[\mathrm{N}$ II $]$ apparent fluxes. Then the [O III] 5007 luminosity 


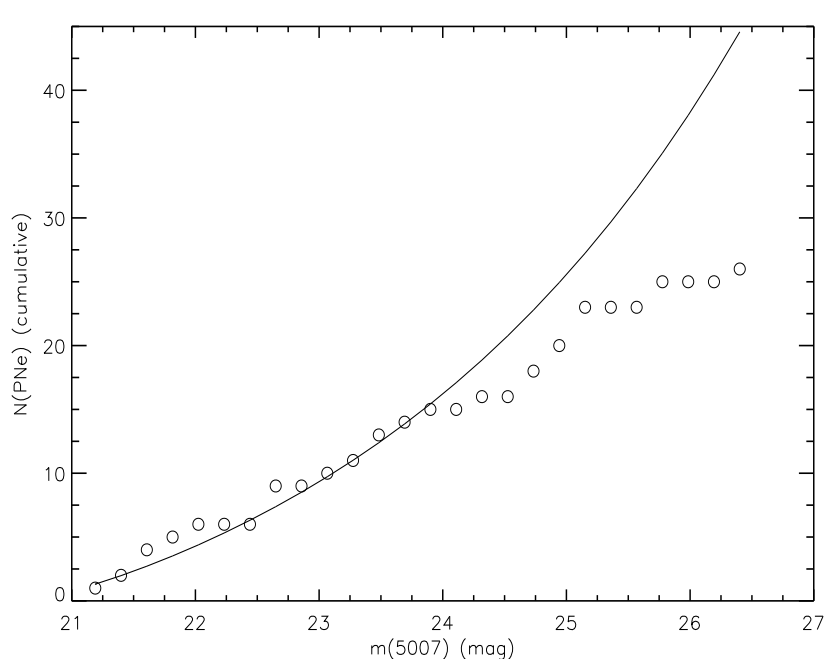

Fig. 5. Cumulative PNLF for NGC 6822 PN candidates (data in Table 2). The fit was calculated for the 4 brightest magnitudes and it returns the product $N \exp (-0.307 \mu)$ and $m_{5007}^{*}$ (see text).

function can be constructed. However, 26 objects is a very small number for reliable statistics in order to derive the peak magnitude of the PNLF and the distance to NGC 6822. On the other hand, the PNLF helps to obtain a better understanding of the PN populations in a galaxy; thus, with this purpose, in the following we will analyze the cumulative PNLF and then the PNLF.

\subsection{The cumulative $P N$ luminosity function}

The cumulative luminosity function can be constructed using apparent magnitudes instead of absolute magnitudes, as Peña et al. (2007) did for the PNe in NGC 3109. We convert the usual luminosity function

$N(M) \propto \exp \left(0.307 M_{5007}\right)\left(1-\exp \left(3\left(M_{5007}^{*}-M_{5007}\right)\right)\right)$

(Jacoby 1989; Ciardullo 1989) to a luminosity function in apparent magnitudes

$$
\begin{aligned}
N(m)= & N \exp (-0.307 \mu) \exp \left(0.307 m_{5007}\right) \\
& \times\left(1-\exp \left(3\left(m_{5007}^{*}-m_{5007}\right)\right),\right.
\end{aligned}
$$

where $N(m)$ is the number of objects brighter than $m, N$ is a normalization constant, $\mu$ is the apparent distance modulus, $\mu=5 \log \mathrm{d}-5+\mathrm{A}_{5007}$, and $M_{5007}^{*}$ and $m_{5007}^{*}$ are the absolute and apparent peak magnitudes of the luminosity function, respectively, the latter defined via

$m(5007)=-2.5 \log F_{5007}-13.74$

(Allen 1973; Jacoby 1989).

In Fig. 5, we show the observed cumulative function for PN data in Table 2. We can see a plateau at the faint end of the observed function, starting at about $25 \mathrm{mag}$. This is probably due to incompleteness of our sample. We have fitted Eq. (2) to the first 4 mag of data using a fitting scheme based on the LevenbergMarquardt technique which employes a $\chi^{2}$ minimization. The errors in the photometry, as given in Table 3, were included in the fit procedure; therefore the brightest magnitudes (with the smallest uncertainties) have higher weights in the fit. The fit has been overlaid on the observed cumulative PNLF in Fig. 5. The best-fit parameters obtained are $m^{*}(5007)=20.47 \pm 0.17$ and $N \exp (-0.307 \mu)=(5.0 \pm 0.6) \times 10^{-3}$. The errors quoted for

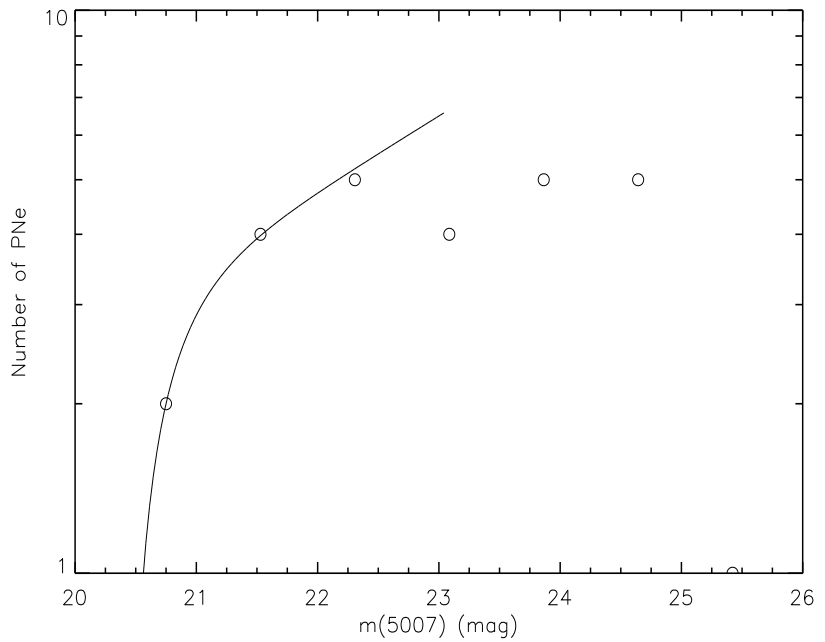

Fig. 6. The luminosity function for PNe in NGC 6822. A bin size of 0.78 mag has been used. The line is our best fit for the three brightest magnitudes. The PNLF shows a dip around 2.5 mag fainter than the brightest PN.

these parameters (given at the three sigma level) correspond to the formal uncertainties of the fitting procedure and they do not represent real error bars because the real errors are correlated. A different statistical procedure including a Monte-Carlo simulation is required to derive real errors (e.g., Hanes \& Whittaker 1987). Such a procedure is beyond the scope of this work.

For the foreground extinction at 5007 towards NGC 6822 we adopted, as in Sect. 3.2, $E(B-V)=0.26 \pm 0.04$ mag which with the Fitzpatrick (1999) reddening law corresponds to $A_{5007}=3.34$ $E(B-V)=0.87 \pm 0.13 \mathrm{mag}$. From this extinction and the distance modulus by Gieren et al. (2006), we derive $M_{5007}^{\star}=-3.71_{-0.42}^{+0.21}$. These errors were obtained from the formal errors given by the fit, the uncertainties in the extinction and the distance modulus by assuming that they add in quadrature. We have also included the possibility that $20 \%$ of the flux has been lost due to the narrow spectroscopic slit (thus objects could be $0.2 \mathrm{mag}$ brighter). The value obtained for $M^{\star}(5007)$ is faint compared to the value -4.08 predicted by Ciardullo et al. (2002) for galaxies with a metallicity similar to the one of NGC 6822, but the error bars are large in both cases and our result agrees with Ciardullo et al. within uncertainties.

\subsection{The PNLF behavior}

From our [O III] 5007 calibrated magnitudes for the PN sample we computed the observed PNLF using a bin size of $0.78 \mathrm{mag}$ which is presented in Fig. 6. In spite of the small number of objects, we are confident that the sample is complete in the 3 brightest magnitudes (a Kolmogorov-Smirnov test was run to verify this, see below).

Interestingly, the observed PNLF seems not to increase monotonically with decreasing luminosity as predicted, but shows a dip at $m(5007) \sim 23$ (about 2.5 mag below the brightest PNe), similar to the one presented by the SMC PNLF (Jacoby \& De Marco 2005). This result has been mentioned by Leisy et al. (2005) with only 17 PN candidates. Our sample includes 9 objects more; nevertheless, we did not find any new bright PNe apart from the ones reported previously (Leisy et al. 2005; Richer \& McCall 2007). Thus, the dip seems statistically significant. To show this we fit the empirical PNLF (Eq. (1)) to the 3 brightest magnitudes (using the procedure given in the 
previous section and including the photometric uncertainties of Table 3) and we run a Kolmogorov-Smirnov test in order to compare the observed PNLF with the empirical PNLF down to 23 mag (including the dip). The test shows that the observed PNLF does not follow the empirical function at the $92 \%$ significance level, meaning that the dip is statistically significant; however this result is not conclusive.

For the dip found in the SMC, Jacoby \& De Marco argued that this could be interpreted as due to the existence of a younger population of PNe in which the central star evolution proceeds very quickly. Such an interpretation could be valid for NGC 6822 $\mathrm{PNe}$, as this galaxy is similar in several ways to the SMC and it seems to possess two stellar components.

As the top 3 mag of the PNLF are significantly complete and this is the distance-sensitive segment, it is tempting to make a rough estimate of the distance to NGC 6822 via the fitting of this segment of the PNLF. From our best fit we derive an apparent peak 5007 mag $m^{*}(5007)=20.43 \pm 0.19$ (error at three sigma). This value should be dereddened for the foreground extinction. As before, we used $A_{5007}=0.87 \pm 0.13 \mathrm{mag}$. Then, by adopting a peak absolute magnitude $M^{\star}(5007)=-4.08$ (as derived from expression (3) of Ciardullo et al. 2002, for the NGC 6822 metallicity), we obtain a distance modulus $m-M=23.64_{-0.43}^{+0.23}$. The error bars include the formal error of the fit, the error in the reddening and, on the low side, a $20 \%$ error due to possible flux losses in the spectroscopic slit has been added. Our derived distance is in agreement, within uncertainties, with the distance reported from Cepheids, e.g., $23.31 \pm 0.02$ by Gieren et al. (2006).

\subsection{Computing $\alpha_{0.5}$}

An interesting parameter that can be estimated once the PNLF is known is $\alpha$, the number of PNe normalized to the parent-galaxy bolometric luminosity. Given the incompleteness of the PNLF at a few magnitudes below the maximum, Ciardullo et al. (2005) suggest computing $\alpha_{0.5}$, that is, the ratio of PNe in the 0.5 brightest magnitude to the bolometric luminosity.

From our observed PNLF, which we have determined it is complete in the first $2.5-3 \mathrm{mag}$, we find that the number of PNe in the first 2 mag is $6_{-0.5}^{+1.0}$ objects. According to the integral PNLF there should be 9 times more PNe in the top 2 mag than in the top $0.5 \mathrm{mag}$. Therefore the number of $\mathrm{PNe}$ in the brightest $0.5 \mathrm{mag}$ is $0.67_{-0.06}^{+0.11}$.

The bolometric luminosity can be estimated from the absolute visual magnitude and by assuming a bolometric correction according to the galactic type (Buzzoni et al. 2006). From Mateo (1998) we adopted the visual integrated apparent magnitude to be $V=9.1 \pm 0.2 \mathrm{mag}$, which can be dereddened with $E(B-V)=0.26 \pm 0.04 \mathrm{mag}\left(A_{V}=3.04 \times E(B-V)=0.79\right)$, giving $V_{0}=8.3 \pm 0.2$. Then the absolute $M_{\mathrm{V}}$ is $-15.0 \pm 0.2$ if the Cepheid distance modulus $23.31 \pm 0.02$ is used. Buzzoni et al. (2006) suggest a mean bolometric correction of -0.8 as representative for any kind of galaxy; in particular for irregular galaxies this value could be slightly smaller, -0.9 (see their Fig. 9). We will adopt $-0.9 \pm 0.1$. Thus the bolometric magnitude for NGC 6822 is about $-15.9 \pm 0.22 \mathrm{mag}$, resulting in a bolometric luminosity $L_{\text {bol }}=\left(1.77_{-0.34}^{+0.41}\right) \mathrm{E} 8 L_{\odot}$.

Thus we obtained $\alpha_{0.5} \sim\left(3.8_{-0.71}^{+0.90}\right)$ E-9. It has been found that $\alpha_{0.5}$ depends upon some properties of the parent galaxy. Galaxies with recent star formation and small galaxies show larger $\alpha_{0.5}$ than early-type galaxies (Ciardullo et al. 2005). The value derived for NGC 6822 is similar to the values derived for small galaxies $\left(M_{\mathrm{B}}\right.$ fainter than -18$)$ and galaxies with recent star formation, which have $\alpha_{0.5} \sim 2.5 \mathrm{E}-9$. In this sense the PN density to bolometric luminosity in NGC 6822 behaves accordingly with its classification as a dwarf irregular.

\section{Conclusions}

Deep [O III 5007 and $\mathrm{H} \alpha+[\mathrm{N}$ II] on-band and off-band imaging have been performed with wide field cameras (in particular CTIO MOSAIC 2) to search for line emission objects in the dwarf irregular galaxy NGC 6822. For some objects we obtained follow-up spectroscopy during a spectroscopic run with VLT FORS 2. Our results are the following:

1) Using several criteria to distinguish between different types of line emission objects, we detected 26 PN candidates (8 more than the previously known sample), many compact HII regions and some $\mathrm{H} \alpha$ emitting stellar objects (the brightest of them could be Ae-Be stars). From our follow-up spectroscopy and using results from the literature, 14 of the $26 \mathrm{PN}$ candidates have been confirmed as true planetary nebulae.

2) We find that compact HII regions and $\mathrm{H} \alpha$ emitting objects are distributed in the central zone of the galaxy where the HI disk found by de Blok \& Walter (2000) also resides as does the young stellar population. No recent star-forming region is found farther than a few kpc from the center. On the other hand, PNe are more widely distributed, with some objects lying in the extreme west and east of the galaxy. This indicates that PNe belong to a different, more widely spread stellar population.

3) The instrumental [O III] 5007 and $\mathrm{H} \alpha+[\mathrm{N} \mathrm{II}]$ magnitudes derived from the imaging were calibrated using results from our spectroscopy. This allowed us to determine calibrated apparent [O III] 5007 and $\mathrm{H} \alpha+[\mathrm{N}$ II] magnitudes.

4) We built the observed cumulative and differential [O III] PNLF. The differential PNLF presents a dip at about $2.5 \mathrm{mag}$ below the brightest magnitude, similar to the one found in the SMC by Jacoby \& De Marco (2002). A KolmogorovSmirnoff test comparing the observed and empirical PNLFs shows that this dip is statistically significant with a $92 \%$ significance level. As in the case of the SMC, the dip can be explained arguing that it could be caused by the presence of two different PN populations where the younger would have central stars evolving very quickly. As NGC 6822 seems to be a galaxy with two stellar components: a HI disk with young population and a spheroidal stellar component, two PN populations could be expected in this galaxy.

5) Our best fit for the 3 brightest magnitudes of the observed PNLF allows us to determine a distance modulus $m-M=$ $23.64_{-0.43}^{+0.23}$ mag which, within uncertainties, is in agreement with recent values from Cepheid stars reported in the literature.

6) From the cumulative PNLF we derived the peak absolute magnitude of the PNLF, $M_{5007}^{\star}=-3.71_{-0.42}^{+0.21}$, which is faint compared to the value -4.08 predicted by Ciardullo et al. (2002) for galaxies with a metallicity similar to the one of NGC 6822, but the error bars are large, and our result agrees with the Ciardullo et al. one within uncertainties. One of the benefits of observing low-metallicity galaxies like NGC 6822 is a better understanding of the absolute magnitudes of their planetary nebulae.

7) We have estimated the number of $\mathrm{PNe}$ in the brightest $0.5 \mathrm{mag}$, normalized to the galactic bolometric luminosity, $\alpha_{0.5}$, to be $\left(3.8_{-0.71}^{+0.90}\right)$ E-9. This value is similar to the values derived for small galaxies $\left(M_{\mathrm{B}}\right.$ fainter than $\left.-18 \mathrm{mag}\right)$ and 

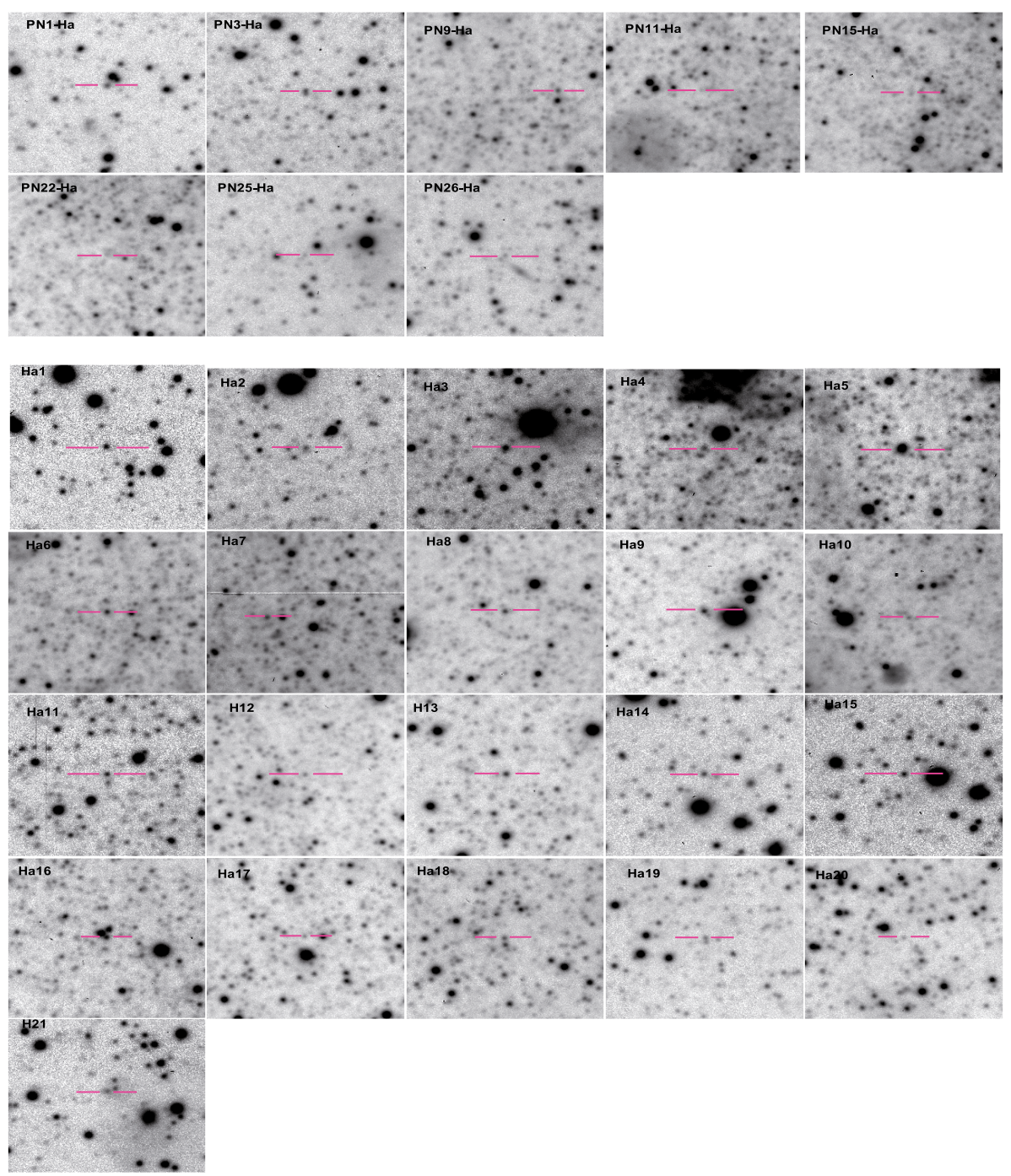

Fig. 7. ID charts for the newly discovered objects. The charts are $30^{\prime \prime}$ per side and were obtained from the $\mathrm{H} \alpha$ image. Each chart is marked with the object number, according to Table 2.

galaxies with recent star formation, and larger than the values obtained for early-type galaxies. Thus the PN density to bolometric luminosity in NGC 6822 behaves accordingly with its classification as a dwarf irregular.

Acknowledgements. Invaluable comments and support by Michael Richer are deeply appreciated. M. Peña is grateful to DAS, Universidad de Chile, for hospitality during a sabbatical stay when part of this work was performed. L. H.-M. benefited from the hospitality of the Departamento de Astronomía, Universidad de Chile. We thank an anonymous referee for her/his comments and suggestions. L. H.-M. received a scholarship from CONACYT-México and DGAPA-UNAM. M. P. gratefully acknowledges financial support from FONDAP-Chile and DGAPA-UNAM. This work received financial support from grants \#43121 (CONACYT-México), IN-114805 and IN-112708 (DGAPA-UNAM).

\section{References}

Allen, C. W. 1973, Astrophysical Quantities (London: Athlone)

Buzzoni, A., Arnaboldi, M., \& Corradi, R. L. M. 2006, MNRAS, 368, 877

Ciardullo, R., Ford, H. C., Neil, J. D., Jacoby, G. H., \& Shafter, A. W. 1987, ApJ, 318,520 .

Ciardullo, R., Jacoby, G. H., Ford, H. C., \& Neill, J. D. 1989, ApJ, 339, 53

Ciardullo, R., Feldmeier, J. J., Jacoby, G. H., et al. 2002, ApJ, 577, 31

Ciardullo, R., Sigurdsson, S., Feldmeier, J. J., \& Jacoby, G. H. 2005, ApJ, 629, 499

de Blok, W. G. J., \& Walter, F. 2000, ApJ, 537, 95 de Blok, W. G. J., \& Walter, F. 2006, AJ, 131, 343

Demers, S., Batinelli, P., \& Artigau, E. 2006, A\&A, 456, 905

Fitzpatrick, E. L. 1999, PASP, 111, 63

Ford, H., Peng, E., \& Freeman, K. 2002, The Dynamics, Structure \& History of Galaxies, ASP Conf. Ser., 273, 41

Gallart, C., Aparicio, A., Bertelli, G., \& Chiosi, C. 1996, AJ, 112, 1950

Gieren, W., Pietrzyński, G., Nalewajko, K., et al. 2006, ApJ, 647, 1056

Hanes, D. A., \& Whittaker, D. G. 1987, AJ, 94, 906

Herrmann, K. A., Ciardullo R., Feldmeir, J. J., \& Vinciguerra, M. 2008, ApJ, 683, 630

Hodge, P., Kennicutt, R. C. Jr., \& Lee, M. G. 1988, PASP, 100, 917

Jacoby, G. H. 1989, ApJ, 339, 39

Jacoby, G. H, \& De Marco, O. 2002, AJ, 123, 269

Killen, R. M., \& Dufour, R. J. 1982, PASP, 94, 444

Lee, H., Skillman, E. D., \& Venn, K. A. 2006, ApJ, 642, 813

Leisy, P., Corradi, R. L. M., Magrini, L., et al. 2005, A\&A, 436, 437

Magrini, L., Corradi, R. L. M., Mampaso, A., \& Perinotto, M. 2000, A\&A, 355, 713

Martins, F., Schaerer, D., \& Hillier, D. J. 2005, A\&A, 436, 1049

Massey, P., Armandroff, T. E., Pyke, R., Patel, K., \& Wilson, C. D. 1995, AJ, 110,2715

Mateo, M. 1998, ARA\&A, 36, 435

Méndez, R. H., Kudritzki, R., \& Herrero, A. 1992, A\&A, 260, 329

Peimbert, A., Peimbert, M., Ruiz, M. T. 2005, ApJ, 634, 1056

Peña, M., Richer, M. G., \& Stasinska, G. 2007, A\&A, 466, 75

Richer, M. G., \& McCall, M. 1995, ApJ, 445, 642

Richer, M. G., \& McCall, M. 2007, ApJ, 658, 328

Valdes, F. G. 1998, Astronomical Data Analysis Software and Systems VII, ed. R. Albretch, R. N. Hook, \& H. A. Bushouse, ASP Conf. Ser., 145, 53 